\title{
Silent hypokalemia - a sometimes fatal condition
}

\author{
Carolina Cadório ${ }^{1,2}$, André Simões ${ }^{1}$, João Gonçalves Pereira ${ }^{1}$ \\ ${ }^{1}$ Intensive Care Unit, Hospital de Vila Franca de Xira \\ 2 Internal Medicine Department, Hospital de Vila Franca de Xira
}

\section{CASE-REPORT}

A 69-year-old Caucasian female was sent to the emergency room by her general practitioner due to severe hypokalemia $(1.7 \mathrm{mmol} / \mathrm{L})$ detected in a routine evaluation.

Her medical history was remarkable only for a 20-year history of hypertension and vertigo two years ago. Her medication included an association of perindopril, indapamide and amlodipine $(10 / 2.5 / 10 \mathrm{mg})$, omeprazole (20mg), lorazepam (1mg) and betahistine $(24 \mathrm{mg})$.

A family history of hypertension (mother, 6 siblings and two sons of 34 and 42 years of age) was present.

She has been experiencing in the previous months increasing hypertension, dry mouth, paresthesia in the lower limbs and weight loss (10 kg during the previous year). No changes in medication was ordered during that time.

In retrospect, it was noted that blood tests, performed three years as well as three months prior to admission already showed hypokalemia (respectively, $3.1 \mathrm{mmol} / \mathrm{L}$ and $2.3 \mathrm{mmol} / \mathrm{L}$ ). At that time point a prolonged QTc interval was also present in the electrocardiogram (EKG).

On admission to the emergency room, her blood pressure was very high, 216/111 mmHg; a systolic murmur was heard at left sternal border and minor hydrostatic peripheral edema was found.

An arterial blood gas sample showed metabolic alkalemia $(\mathrm{pH}$ 7.507, PCO2 48.2 mmHg, PO2 78.3 mmHg, HCO3-37.3 mmol/L). Other laboratory testing revealed an increased serum creatinine of $1.2 \mathrm{mg} /$ $\mathrm{dL}$ (previously $0.8 \mathrm{mg} / \mathrm{dL}$ ), urea $45 \mathrm{mg} / \mathrm{dL}$, natremia $\left(\mathrm{Na}^{+}\right) 142 \mathrm{mmol} / \mathrm{L}$, hypokalemia $\left(\mathrm{K}^{+}\right)$of $2.01 \mathrm{mmol} / \mathrm{L}$, chloride $\left(\mathrm{Cl}^{-}\right)$of $98 \mathrm{mmol} / \mathrm{L}$, magnesium of $2.1 \mathrm{mg} / \mathrm{dL}$. Hemogram was normal (hemoglobin $13.3 \mathrm{~g} / \mathrm{dL}$, platelets $244 \times 10^{9} / \mathrm{L}$ ) and LDH was $307 \mathrm{U} / \mathrm{L}$. The urinary ionogram showed $\mathrm{Na}^{+} 109 \mathrm{mEq} / \mathrm{L}, \mathrm{K}^{+} 19 \mathrm{mEq} / \mathrm{L}, \mathrm{Cl}^{-} 115 \mathrm{mEq} / \mathrm{L}$. Thyroid function tests were normal. The EKG showed sinus rhythm with a prolonged QT associated with flattened T waves.

The patient was admitted to an intensive care unit and started on a continuous intravenous infusion of potassium chloride (15 mEq/h), supplemented with oral potassium chloride and spironolactone, 50 $\mathrm{mg}$ daily. This was supplemented with magnesium sulfate during three days as approximately $50 \%$ of patients with hypokalemia also have concomitant magnesium deficiency. Although approximately $99 \%$ of the body's total store of magnesium lies intracellular, a normal serum magnesium may grossly underestimate a true body deficit of magnesium, so magnesium replacement should be considered for patients with hypokalemia. ${ }^{1}$ Progressive normalization of serum $\mathrm{K}^{+}$ concentration as well as of EKG was noted.

Further investigation was pursued with blood tests and an abdominal computed tomography (CT), which was unremarkable except for a nodular lesion, measuring roughly $3 \mathrm{~cm}$, in the right adrenal gland, that was suggestive of adenoma.

\section{WHAT IS THE MOST COMMON CAUSE OF SECONDARY HYPERTENSION?}

Primary hypertension accounts for $90-95 \%$ of adult cases and may occur as a result of environmental or genetic causes. Secondary hypertension, although rare, has multiple etiologies, making its correct investigation and classification more difficult. However, identifying the underlying cause makes achieving a cure more likely.

Every physician should be alert to the possibility of secondary hypertension. Clinical suspicion should be based on patient age at onset, presence of malignant or accelerated hypertension, and an acute rise in blood pressure from previously stable readings.

Our patient was a 67-year-old female. The most common cause of secondary hypertension in this age group ( $>65$ years) is renal artery stenosis and renal failure, secondary to atherosclerosis. However, this patient had had uncontrolled hypertension over the previous 10 years; therefore, her differential diagnosis could be broadened to include diagnoses more common to a younger age group ( 40 to 64 years). At that age, hyperaldosteronism, obstructive sleep apnea, Cushing's syndrome and pheochromocytoma are more frequent. 2,3

From the clinical history, obstructive sleep apnea was excluded as the patient has no history of snoring, nocturne apneic episodes or daytime sleepiness. Pheochromocytoma was a weak hypothesis, as the patient had no suggestive clinical symptoms. Nevertheless, plasmatic metanephrines were checked and found to be normal (normetanephrines $78 \mathrm{pg} / \mathrm{mL}$, metanephrines $73 \mathrm{pg} / \mathrm{mL}$ ).

On physical examination, the patient had no signs of Cushing's syndrome, such as buffalo hump, central obesity or moon facies. Further laboratory investigation also showed normal 24-hour urinary free cortisol (28 ug/24h), ACTH (13.3 pg/mL), morning salivary cortisol $(5.38 \mathrm{nmol} / \mathrm{L})$ and serum cortisol $(12.5 \mu \mathrm{g} / \mathrm{dL})$.

We excluded renal artery stenosis with a CT angiography. Consideration for chronic renal failure was discarded since the patient had 
previously normal serum creatinine, normal renal measurement, low urine albumin (3.7 mg/L). Moreover, the albumin to creatinine urinary ratio was also low: $14.6 \mathrm{mg} / \mathrm{g}$.

Primary hyperaldosteronism (PA) seemed the most probable diagnosis, since the patient presented with hypokalemia, metabolic alkalemia and hypertension.

PA is the most common cause of secondary hypertension and consists of an inappropriately elevated aldosterone production, in the setting of low plasma renin. The aldosterone, by inducing renal reabsorption of sodium at the distal convoluted tubule, increases secretion of $\mathrm{K}^{+}$and hydrogen $\left(\mathrm{H}^{+}\right)$ions, causing hypernatremia, hypertension, hypokalemia and alkalosis. 2

The major forms of hyperaldosteronism are aldosterone-producing adenoma and bilateral idiopathic adrenal hyperplasia. Other entities are familial forms of primary aldosteronism, ectopic secretion of aldosterone (more common in ovaries or kidneys) and pure aldosterone-producing adrenocortical carcinomas. This pathology is often underdiagnosed and undertreated, which leads to cardiovascular morbidity and also mortality. This underlines the importance of an early diagnosis and treatment. ${ }^{4}$

The most recent indications for PA screening include patients with hypertension resistant to 3 conventional antihypertensive drugs; hypertension with hypokalemia (spontaneous or diuretic induced); hypertension and a family history of either early-onset hypertension or cerebrovascular accident at a young age ( $<40$ years); hypertension and an adrenal tumor; controlled blood pressure on $\geq 4$ antihypertensive drugs; sustained blood pressure $>150 / 100 \mathrm{mmHg}$, measured on 3 different days; hypertension and sleep apnea, and all hypertensive first-degree relatives of patients with primary aldosteronism. Our patient meets at least 3 of these criteria. ${ }^{3,4}$

Given the strong positive family history Liddle's syndrome should be considered as a differential diagnosis However, our patient presented a high plasmatic aldosterone and normal urinary sodium which allowed us to exclude this diagnose.

The hypothesis of thrombotic microangiopathy was rejected since the patient had normal platelet count, normal hemoglobin and no raised $\mathrm{LDH}$.

Based on ESC/ESH guidelines for the management of arterial hypertension, hypertensive emergency is defined by the presence of organ damage. In this case report, even if asymptomatic, our patient presented with a CKD eGFR of $47 \mathrm{ml} / \mathrm{min} / 1.73 \mathrm{~m} 2$ that should have raised suspicion of a hypertension emergency, and therefore should have undergone fundoscopy examination. ${ }^{5}$

\section{WHY DOES THIS PATIENT HAVE HYPOKALEMIA AND METABOLIC ALKALOSIS?}

As described above, in PA the high circulating levels of aldosterone will act in the distal convoluted tubule and cortical collecting duct of the kidney. This promote the expression of amiloride-sensitive epithelial sodium channels $(\mathrm{ENaC})$ in the distal tubule and cortical collecting duct, resulting in sodium reabsorption to the intracellular compartment, which is then sent to the blood through interstitial fluid via $\mathrm{Na}+/ \mathrm{K}+$ ATPase pumps on the basolateral surface of the cells, in trade of $\mathrm{K}^{+}$that is secreted into urine via the apical renal outer medullary $\mathrm{K}^{+}$channel (ROMK) and causing the hypokalemia. Aldosterone also stimulates the urinary secretion of $\mathrm{H}^{+}$via the $\mathrm{H}^{+}$-ATPase in the intercalated cells of the cortical collecting tubules, causing the alkalosis. $^{4}$

\section{WHAT IS THE MOST LIKELY CAUSE OF HYPOKALEMIA AND HYPERTENSION?}

Hypokalemia is a common clinical problem that can result from decreased intake, increased translocation into the cells or, most often, increased losses of $\mathrm{K}^{+}$in the urine or in the gastrointestinal tract.

Urinary $\mathrm{K}^{+}$wasting is characteristic of any condition associated with primary hypersecretion of a mineralocorticoid, such as an aldosterone-producing adrenal adenoma. ${ }^{6}$

Consequently, PA PA differential diagnoses include excess diuretic use including furosemide [inhibiting the $\mathrm{Na}^{+} \mathrm{K}^{+} 2 \mathrm{Cl}^{-}$cotransporter (NKCC2) in the thick ascending limb] and thiazides [inhibiting the $\mathrm{Na}^{+} \mathrm{Cl}^{-}$ cotransporter (NCC) in the distal convoluted tubule]. These drugs, by inhibiting more proximal $\mathrm{Na}^{+}$reabsorption, increase its delivery to the more distal connecting tubule and collecting duct where the ENaC-mediated electrogenic $\mathrm{Na}^{+}$transport creates an electrical driving force for $\mathrm{K}^{+}$secretion via the ROMK $\mathrm{K}^{+}$channel. Further, they cause volume depletion and indirectly activate the renin-angiotensin-aldosterone system. A differential diagnosis with excessive excretion of nonreabsorbable anions, such as bicarbonate or beta-hydroxybutyrate, and renovascular disease, in which increased secretion of renin leads to enhanced aldosterone release, should also be considered. $4,6,7$

In this clinical case, despite the patient taking a thiazide diuretic, urinary $\mathrm{K}^{+}$loss induced by diuretics was not corroborated by the urinary ionogram, which showed urinary $\mathrm{K}^{+}$in the normal range.

As mention above, PA is the most common cause of secondary hypertension, with a prevalence of $20 \%$ among patients with resistant hypertension. The presence of primary mineralocorticoid excess should be suspected in any patient with the triad of hypertension, unexplained hypokalemia, and metabolic alkalosis.

\section{IS IT POSSIBLE FOR A PATIENT TO HAVE NORMAL SERUM POTASSIUM AND PA? SHOULD WE TRUST ARR (PLASMA ALDOSTERONE TO RENIN RATIO) AS A MEANS OF DIAGNOSIS?}

Hypokalemia is considered to be one of the major clinical features of PA. However, most patients with primary mineralocorticoid excess are normokalemic - only about $40 \%$ of patients with PA have hypokalemia. This is likely related to earlier diagnosis, as more patients with hypertension are being screened with the plasma aldosterone to renin ratio (ARR), as a case-detection test for primary aldosteronism. ${ }^{3}$ 
For the diagnostic work-up, some experts suggest first identifying a low plasma renin activity (PRA $<1 \mathrm{ng} / \mathrm{mL} / \mathrm{h})$ and then requesting an aldosterone test (which must be increased $>10 \mathrm{ng} / \mathrm{dL}$ ). An ARR $>20$ is commonly used as the threshold for positive PA screening, with a sensitivity of $78 \%$ and a specificity of $83 \%$ in patients with resistant hypertension. The most important pitfall of PA screening is that some laboratories report PRA values as low as $0.1 \mathrm{ng} / \mathrm{mL} / \mathrm{h}$. Accordingly, an $A R R>20$ will be present even when the plasma aldosterone is as low as $2 \mathrm{ng} / \mathrm{dL}$. Therefore, caution is necessary when interpreting an isolated ARR, especially when PRA is $<0.6 \mathrm{ng} / \mathrm{mL} / \mathrm{h}$. Another important consideration for a diagnosis of PA is that ARR and PRA must only be measured with a normal serum $\mathrm{K}^{+}$, as hypokalemia can inhibit aldosterone production and cause a false negative test. ${ }^{3}$

There are other pitfalls related to the screening of PA, such as antihypertensive medication (diuretics, angiotensin-converting enzyme inhibitors, and angiotensin receptor blockers can all cause false negative results), or misinterpretation of laboratory values (such as using direct renin concentration instead of PRA to calculate ARR). If the clinical suspicion is high, ARR should be repeated after withdrawing these antihypertensive medications for at least two weeks. ${ }^{3}$

We described a female patient with a 10-year history of drug-resistant hypertension, metabolic alkalosis and severe hypokalemia, first identified in blood tests performed 3 years before the admission. Despite patient having an extremely low value of serum $\mathrm{K}^{+}$, no significant clinical features such as muscle weakness, constipation or fatigue were noted. Moreover, she had no other major electrolyte imbalances, including excessive urinary $\mathrm{K}^{+}$excretion or hypernatremia. Accordingly, we believe that this clinical scenario had a long evolution and some degree of adaptation. In fact, our patient presented with normal kaliuresis, despite the use of indapamide and the PA. The authors cannot exclude a concomitant mechanism of $\mathrm{K}$ loss, namely gastrointestinal (covert vomiting), but there was no clinical reference to this aspect.

Patient's blood tests showed low plasmatic renin activity (PRA; $0.13 \mathrm{ng} / \mathrm{mL} / \mathrm{h}$ ), high plasmatic aldosterone concentration (PAC, 34 $\mathrm{ng} / \mathrm{dL}$ ) and an ARR of 23, which is, in this clinical scenario, confirmatory of PA. It must be remembered that blood samples were collected during severe hypokalemia correction, but this should lead to lower than normal aldosterone activity (and not to these high levels).

As PA was suspected, the patient underwent an adrenal CT scan, which confirmed a right adrenal node of $3 \mathrm{~cm}$ diameter suggestive of adenoma. Our final diagnosis was PA.

It should be remembered that an isolated adrenal node in a CT scan is hardly a diagnosis of PA; the most commonly identified adrenal nodes are non-functional adrenal adenomas (sometimes referred to as "incidentalomas"), which may be present in up to $4 \%$ of the general population.

\section{DOES PRIMARY HYPERTENSION HAVE THE SAME IMPACT AS HYPERTENSION IN PA?}

This 69-year-old female patient had a 20-year history of primary hypertension (age at diagnosis 50 years old), and, at least during the prior 10 years, had poorly controlled blood pressure and was medicated with three different classes of antihypertensive drugs, including one diuretic. Accordingly, when an acute change in a chronic disease is noted, it should foster an effort to identify its trigger, to adjust the medication and to look for secondary causes of hypertension. Clinical inertia may have undesired clinical consequences on the patient's morbidity and mortality.

In fact, evidence suggests that PA amplifies cardiovascular morbidity and mortality beyond primary hypertension, even after controlling for blood pressure values. Some studies have found that death from cardiovascular causes is more common among patients with PA compared with matched control patients with primary hypertension. ${ }^{3}$

Some pathophysiologic changes are seen in PA patients' autopsies, such as fibrosis of the heart, adrenal glands, pancreas, and lungs. It has been found that PA is associated with vascular remodeling characterized by a marked increase in arterial media/lumen ratio. These changes occur even in normotensive individuals. Stowasser et al. studied a population of normotensive patients with PA and found higher rates of concentric left ventricular hypertrophy and poor diastolic function compared with age and sex matched normotensive control participants. Milliez et al. also found that, compared with patients with primary hypertension, patients with PA were more likely to have stroke, nonfatal myocardial infarction, or atrial fibrillation. ${ }^{3}$

\section{WHAT ABOUT PATIENTS WHO SCREEN NEGATIVE FOR PA?}

The prevalence of PA in patients with resistant hypertension might be as high as $20 \%$. Moreover, some patients with negative screen tests for PA might still have a mild form of the disease, not meeting criteria for a formal diagnosis, yet possibly contributing to their hypertension and long-time morbidity.

For this group of patients with resistant hypertension, spironolactone is recommended, especially when a low $\mathrm{K}^{+}$serum level is found. The PATHWAY-2 study demonstrated the superiority of spironolactone to bisoprolol or doxazosin, added to existing regimens, except in patients with the highest direct renin concentration. We recommend treatment with a mineralocorticoid receptor antagonist (MRA) for patients with resistant hypertension, who meet the criteria for PA screening and whose renin is normal or low but whose aldosterone is not high enough for a positive PA diagnosis. Close monitoring of electrolytes is essential when MRA drugs are used. ${ }^{3}$

In this clinical case, our patient was discharged from hospital, receiving lisinopril $20 \mathrm{mg}$, amlodipine $5 \mathrm{mg}$ and spironolactone $100 \mathrm{mg}$. At that time she had achieved adequate blood pressure control and serum potassium normalization. An endocrinology appointment was arranged.

\section{CONCLUSION}

We present a case of a 69-year-old patient with a 10-year history of drug-resistant hypertension. Although her relatively advanced age 
was unusual for secondary hypertension, her clinical features posed a strong suspicion of PA. The fact that she was mostly asymptomatic was suggestive of a long-term evolution of PA and a delay in diagnosis. In fact, she had had known hypokalemia for at least three years.

In patients presenting with progressive resistant hypertension and electrolyte disturbances, screening for secondary hypertension should be performed independent of patient age.

Disclosure of potential conflicts of interest: none declared

\section{References}

1. Mattsson C, Young WF. Primary aldosteronism: Diagnostic and treatment strategies. Nat Clin Pract Nephrol. 2006; 2(4):198-208

2. Charles L, Triscott J, Dobbs B. Secondary hypertension: Discovering the underlying cause. Am Fam Physician. 2017; 96(7):453-61

3. Viera AJ, Neutze DM. Diagnosis of secondary hypertension: An age-based approach. Am Fam Physician. 2010; 82(12):1471-78

4. Byrd JB, Turcu AF, Auchus RJ. Primary aldosteronism. Circulation. 2018; 138(8):823-35

5. Whelton PK, Carey RM, Aronow WS, Casey DE, Collins KJ, Dennison Himmelfarb C, et al. 2017 ACC/AHA/AAPA/ABC/ACPM/AGS/APhA/ASH/ASPC/NMA/PCNA Guideline for the Prevention, Detection, Evaluation, and Management of High Blood Pressure in Adults: Executive Summary. J Am Coll Cardiol. 2018; 71(19):2199-269

6. Kardalas E, et al. Hypokalemia: A clinical update. Endocr Connect, 2018; 7(4):R135-46

7. Ashurst J, Sergent SR, Sergent BR. Evidence-based management of potassium disorders In the emergency department. Emerg Med Pract. 2016; 18(11):1-24

\section{Correspondence to:}

Carolina Cadório, MD

Internal Medicine Department, Hospital de Vila Franca de Xira

E-mail: carolinacadorio@gmail.com 